The demonstration of small left-to-right intracardiac shunts may present a difficult problem during cardiac catheterization; oximetry is seldom diagnostic and a more sensitive technique is required. Various indicator methods have been employed for this purpose; of these the hydrogenplatinum electrode system is extremely sensitive, does not require expensive apparatus, and avoids the risks of left heart catheterization for the injection of indicator or contrast medium. Since its introduction by Clark and Bargeron (1959), several favourable reports have appeared (Vogel, Grover, and Blount, 1962; Arcasoy, Guntheroth, and Mullins, 1962; Hyman et al., 1961). However, this technique has not been adopted widely, nor have accounts of its value been published in this country. We report our experience using this method in the investigation of 50 patients.

\section{Principle, Method, and Patients}

Hydrogen, introduced by inhalation, is detected in the blood-stream by a platinum electrode catheter. Contact of hydrogen with the electrode causes an alteration of potential, which is shown by displacement of the baseline of an intracardiac electrocardiograph.

The patient inhales a single breath of hydrogen from an anaesthetic rebreathing bag, using a face mask, and the time of inhalation is recorded. The electrode, blackened by electrolysis with a 5 per cent solution of platinic chloride (Clark and Bargeron, 1959), is connected to the " $V$ " lead of an electrocardiogram. The electrode may be placed in the pulmonary artery, the right ventricle, the right atrium, and a vena cava. The paper speed of the machine is reduced to $5 \mathrm{~mm}$./sec., and its sensitivity attenuated 10 times.

The inhaled hydrogen, absorbed by pulmonary capillary blood, is returned to the left atrium and ventricle, and, in the absence of a left-to-right shunt, circulates through the systemic vessels, to be returned to the right atrium. This transport of hydrogen from

Received August 2, 1967. the lungs to the right heart requires 8-12 seconds in a normal adult, but this duration may be considerably diminished in a child. This "circulation time" is practically identical wherever the platinum electrode is positioned in the right heart.

In the presence of a left-to-right intracardiac shunt, however small, a proportion of the inhaled hydrogen will cross from the left to the right heart. In a chamber at the level of, or beyond, the defect a rapid deflection of the intracardiac electrocardiograph will occur within 3 seconds of inhalation. However, in that part of the right heart proximal to the shunt the normal delayed circulation curve will be recorded. In a ventricular septal defect, for example, hydrogen would be detected in the right atrium after the normal systemic circulation, and the shift of the hydrogen potential curve would be delayed; on the other hand, hydrogen will circulate rapidly to the right ventricle and to the pulmonary artery (Fig.).

This method was used in the investigation of 50 patients; 2 were infants, 31 were between 2 and 15 years, and 17 were adults. The technique was first used in 21 patients with known shunts in order to assess its reliability. It was then used in 29 patients to detect or exclude a shunt where the results of oximetry were equivocal.

\section{RESULTS}

In the 21 patients with known shunts, 12 had ventricular septal defects, 7 had atrial septal defects, and 2 had patent ductus arteriosus. In every case an early large deflection was obtained at the level of, and distal to, the shunt. Because of the size of the shunt in these cases, the sensitivity of the electrocardiogram had to be attenuated more than 10 times in order to contain the curves.

Seven patients were investigated in whom a small left-to-right shunt was suspected clinically. Five were thought to have ventricular septal defects; one a patent ductus arteriosus; and one, who had had an atrial septal defect repaired, was considered to have a residual or additional shunt. At catheterization the defects were not crossed by the catheter and 


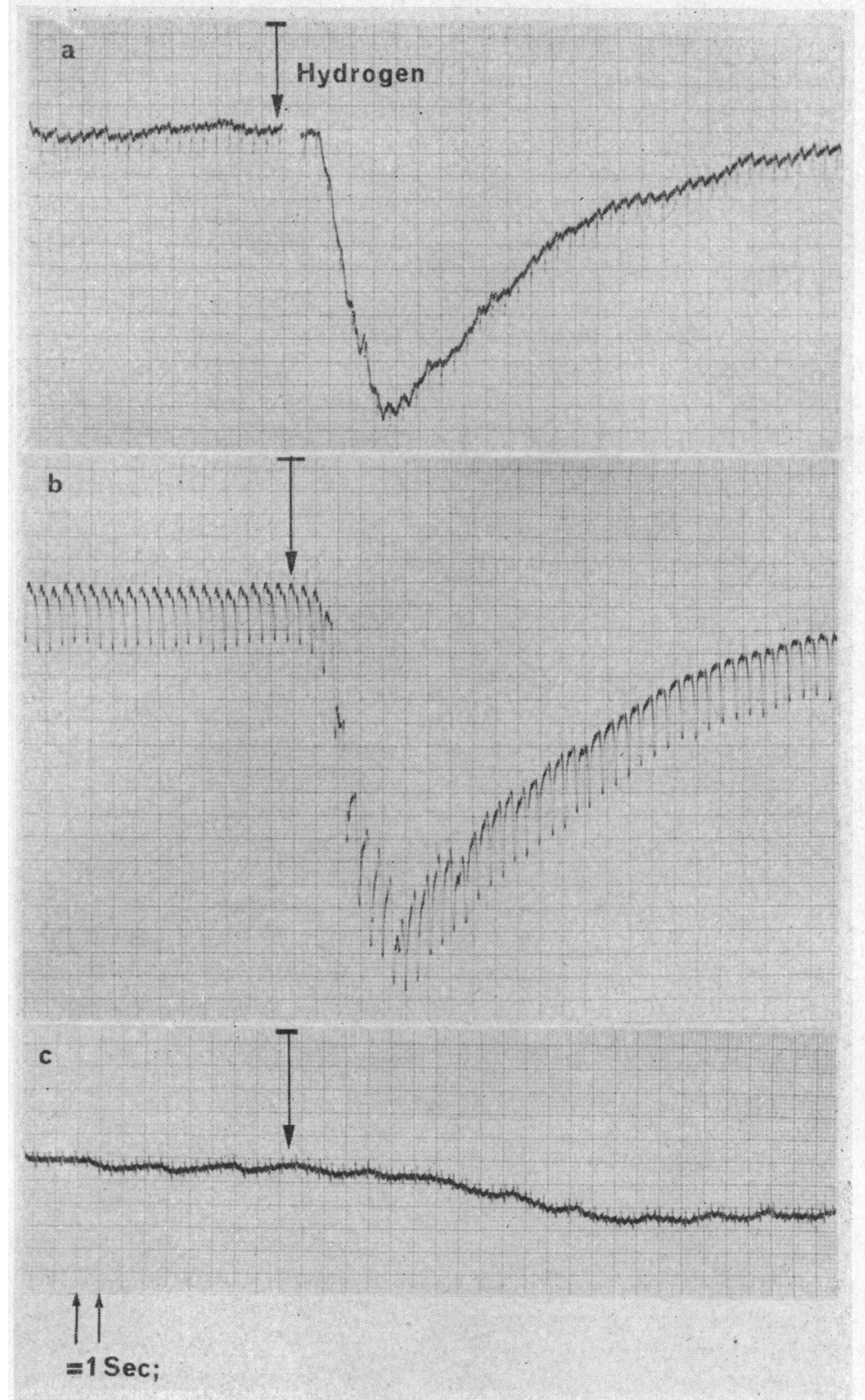

FIG.-Hydrogen curves taken from (a) pulmonary atery; (b) right ventricle; and (c) right atrium, in a patient with a ventricular septal defect which was not demonstrated by oxygen saturations (see text).

oximetry was not diagnostic in any of the patients in this group. Several series of blood samples were taken as rapidly as possible throughout the right heart and the oxygen saturations were measured by a Kipp haemoreflector. In none was there a consistent increase in oxygen saturation within the level of experimental error. In every case, however, the hydrogen curves demonstrated unequivocally the presence and site of the shunt.

In 14 patients the clinical diagnosis was pulmonary stenosis, but an additional atrial septal defect was suspected. At catheterization the atrial septum was patent in most of them; however, negative hydrogen curves excluded a shunt. This was 
considered sufficient evidence to differentiate a patent foramen ovale from a small atrial septal defect.

Four patients had the clinical signs suggestive of ventricular septal defect. Hydrogen curves, however, excluded a shunt; other investigations were then undertaken, which established the diagnosis of mitral regurgitation in three and isolated tricuspid regurgitation in the other.

This technique was also found to be satisfactory for the detection of small bidirectional shunts in association with pulmonary hypertension. Even the slight mixing which takes place near a defect through which the flow is predominantly right to left was detectable by hydrogen curves. Conversely, the absence of a shunt by this method in a pulmonary hypertensive subject is probably sufficient to exclude the Eisenmenger syndrome. In this series there were 4 patients with pulmonary hypertension; in 2 a shunt was present (patent ductus arteriosus and ventricular septal defect), and in 2 it was excluded.

The site of a moderate or large shunt is seldom in doubt clinically. However, oximetry commonly shows a greater rise in oxygen saturation in the chamber distal to the actual shunt. In one patient in this series, difficulty arose in differentiating a ventricular septal defect with aortic regurgitation, from an aorto-pulmonary window. The oxygen saturation rise was detected in the pulmonary artery but not in the right ventricle. However, hydrogen curves established that the shunt was at ventricular level, and the former diagnosis was established and later confirmed at operation.

This method also established the site of an arteriovenous fistula in a patient who presented with a continuous murmur over the back of the chest. Hydrogen curves detected the shunt low in the right atrium and in the coronary sinus, establishing the diagnosis of coronary arteriovenous fistula. The validity of this observation had been tested by obtaining hydrogen curves from the coronary sinus in several patients without shunts, when it was found the curves so produced were similar to those in the vena cava and quite unlike those with a left-to-right shunt.

\section{Discussion}

The hydrogen-platinum electrode system combines the attributes of an efficient and simple method of introducing the indicator into the blood returning to the left side of the heart, with a highly sensitive detector in the right heart. The only other similar techniques involve the use of radioactive gas, which requires infinitely more expensive apparatus and time-consuming management. By contrast, no additional recording apparatus is required for the hydrogen method, since use can be made of the monitoring electrocardiogram. The ease with which satisfactory curves can be obtained is such that catheterization time is only slightly prolonged, and furthermore the patient suffers no discomfort. The sensitivity of this method was clearly seen in the 7 patients who had small shunts. In view of this sensitivity, shown by the large deflections produced in the hydrogen curves, it can be assumed that negative curves exclude a shunt. The value of this technique in localizing the site of shunts is also well illustrated in our series.

Before the introduction of the hydrogenplatinum electrode into our catheter laboratory, we found that the most satisfactory way of demonstrating and localizing small shunts was by angiography of the left heart. The risks of this procedure, the time taken to obtain the high quality films that are necessary to visualize small shunts, and the cost, compare unfavourably with the hydrogen-electrode system.

The inflammable nature of hydrogen gas is the single slight disadvantage of this method. Dickerson, Jensen, and Hollison (1965) using a "Standard Mine Safety Appliance Explosimeter" clearly showed that no explosive risk, with allowances for a liberal safety factor, existed outside a conical envelope, $91 \mathrm{~cm}$. ( $3 \mathrm{ft}$.) above, $15 \mathrm{~cm}$. ( $6 \mathrm{in}$.) below, and $30 \mathrm{~cm}$. (12 in.) lateral to the patient's mouth, and because of the rapid diffusibility of hydrogen the duration of this danger was 25 seconds. Provided, therefore, that simple precautions are taken, the risk of explosion of the hydrogen gas can be abolished, and does not constitute a barrier to the general acceptance of the method.

\section{SUMMARY}

The hydrogen platinum electrode system for the detection of intracardiac shunts was used in the investigation of 50 patients. Initial experience in patients with known shunts demonstrated the ease with which the method can be instituted, and its reliability. The sensitivity of the technique was such that small shunts were detected where results of oximetry were negative. Intracardiac shunts could also be confidently excluded by this method. The explosive risk of the hydrogen can be abolished by simple precautions and does not preclude its general acceptance.

We wish to thank Drs. Wallace Brigden and Lawson McDonald for permission to investigate their patients and for their help in the preparation of this paper, and Messrs. W. Dicks, and G. Mullineux for their technical assistance. 


\section{REFERENCES}

Arcasoy, M. M., Guntheroth, W. G., and Mullins, G. L (1962). Simplified intravascular hydrogen electrode method. Amer. F. Dis. Child., 104, 349.

Clark, L. C., and Bargeron, L. M. (1959). Left-to-right shunt detection by an intravascular electrode with hydrogen as an indicator. Science, 130, 709.

Dickerson, R. B., Jensen, W. L., and Hollison, R. V. (1965).
The safety of hydrogen in shunt detection. Circulation, 31, 705.

Hyman, A. L., Hyman, E. S., Quiroz, A. C., and Gantt, J. R. (1961). Hydrogen-platinum electrode system in detection of intravascular shunts. Amer. Heart f., 61, 53.

Vogel, J. H. K., Grover, R. F., and Blount, S. G. (1962). Detection of the small intracardiac shunt with the hydrogen electrode; A highly sensitive and simple technique. Amer. Heart F., 64, 13. 